\section{Almost periodic parameters for the second moment stability of linear stochastic systems}

\author{
Alessandro N. Vargas and João B. R. do Val
}

\begin{abstract}
The paper presents conditions to assure the uniform second moment stability for a class of linear time-varying stochastic systems. The system parameters are assumed to be almost periodic, a concept that is weaker than the periodic one. Under the existence of the long-run average cost associated with the stochastic system, we apply the almost periodicity to prove the desired stability result. An application illustrates the usefulness of the approach by implementing an almost periodic state-feedback strategy to control the velocity of a DC motor device.
\end{abstract}

\section{INTRODUCTION}

Consider a time-varying discrete-time stochastic linear system defined in a filtered probability space $\left(\Omega, \mathcal{F}, \mathcal{F}_{k}, P\right)$ as follows.

$$
\mathcal{S}:\left\{\begin{array}{l}
x_{k+1}=A_{k} x_{k}+B_{k} w_{k}, \\
y_{k}=C_{k} x_{k}+D_{k} w_{k}, \quad k \geq 0, \quad x_{0} \in \mathbb{R}^{n},
\end{array}\right.
$$

where $x_{k}, w_{k}$, and $y_{k}, k=0,1, \ldots$ are processes taking values respectively in $\mathbb{R}^{n}, \mathbb{R}^{m}$, and $\mathbb{R}^{p}$, which represent the system state, additive noisy input, and output, in this order. The noisy input $\left\{w_{k}\right\}$ forms an independent and identically distributed process with zero mean and covariance matrix equal to the identity for each $k \geq 0$. The matrix sequences $\left\{A_{k}\right\},\left\{B_{k}\right\},\left\{C_{k}\right\}$, and $\left\{D_{k}\right\}$ are known and bounded, with values corresponding to the dimensions $n \times n, n \times m, p \times n$ and $p \times m$, respectively.

Many results appeared in the last decades for settling stability concepts of time-varying linear systems, see for instance landmark results in [1], [2] [3], [4], [5], [6], [7]; see also [8], [9] for an account on some recent extensions for linear parameter time-varying systems.

Stability for stochastic systems is a established theme of investigation, and some pioneering works had settled the current basis [10], [11], [12], [13]; see [14] for recent results. A classical stability concept for stochastic systems, which will be studied in details in this paper, is known as uniform second moment stability and is defined as follows.

Definition 1.1: [10, p.97-98]. The stochastic system $\mathcal{S}$ is called uniformly second moment stable if there exists a constant $c>0$ such that $\mathrm{E}\left[\left\|x_{k}\right\|^{2}\right] \leq c$ for all $k \geq 0$.

The correspondence between the classical uniform second moment stability and the existence of the long-run average cost

Research supported in part by the Brazilian agencies FAPESP grants 03/06736-7 and 04/06947-0, and CNPq grants 304856/2007-0 and 471557/2009-9; by the Spanish agency Fundación Carolina - Programa "Movilidad de profesores e investigadores Brasil-España. C.2010"; and by the Basque Center for Applied Mathematics - "BCAM Visiting fellow program".

Alessandro N. Vargas is with Universidade Tecnológica Federal do Paraná, UTFPR, Av. Alberto Carazzai 1640, 86300-000 Cornélio ProcópioPR, Brazil, and with Basque Center for Applied Mathematics, BCAM, Alameda de Mazarredo 14, E-48009 Bilbao, Vizcaya, Spain. E-mail: avargas@utfpr.edu.br. João B. R. do Val is with Universidade Estadual de Campinas, UNICAMP, Fac. de Engenharia Elétrica e de Computação, C.P. 6101, 13081-970 Campinas-SP, Brazil. E-mail: jboscoddt.fee.unicamp.br is a provocative subject and many aspects of this relation are still open. For instance, it is simple to prove that the uniform second moment stability of $\mathcal{S}$ implies in the existence of the long-run average cost

$$
J=\limsup _{N \rightarrow \infty} \frac{1}{N} \sum_{k=0}^{N-1} \mathrm{E}\left[\left\|y_{k}\right\|^{2}\right],
$$

where $\mathrm{E}[\cdot]$ denotes the mathematical expectation operator and $\|\cdot\|$ represents the Euclidean norm. The converse statement, i.e., that the existence of (1) implies in the uniform second moment stability of $\mathcal{S}$, is false as shown by the counterexample in [15, Ex. 3.1]. The search for conditions to turn this converse statement valid motivates our study. In fact, our approach expands on [15] by investigating the intrinsic properties of the parameters $A_{k}, k \geq 0$, as we explore in the sequence.

A reliable way to confirm the desired stability for $\mathcal{S}$ is to employ the result from [15, Lem. 3.1] (see Proposition 4.3 in connection), which states that if the pair $\left(A_{k}, B_{k}\right)$ is controllable (Definition 2.1), then the system $\mathcal{S}$ is uniformly second moment stable if and only if there exist real numbers $\gamma \geq 1$ and $0<\xi<1$ such that

$$
\left\|A_{k+\ell} \cdots A_{k}\right\| \leq \gamma \xi^{\ell}, \quad \forall k, \ell \in \mathbb{N} .
$$

Thus, the exponential decay in (2) turns to be the key for proving the uniform second moment stability for $\mathcal{S}$.

A preliminary idea to get (2) is to verify whether the parameter matrix sequence $\left\{A_{k}\right\}$ is periodic, i.e., if there is a number $p \in \mathbb{N}$ such that $A_{k+p}-A_{k}=0$ for all $k \in \mathbb{N}$. In the positive case, the exponential decay in (2) follows [15, Cor. 3.2].

A challenging question is to determine whether the exponential decay in (2) remains valid if we replace the "periodic" condition by the weaker notion of "almost periodic" (for a precise definition of almost periodicity, see Definition 3.1). The answer is not immediate because, for continuoustime systems, there are linear equations with almost periodic parameters for which the solution is asymptotically stable but not uniformly stable, see [16], [17, p.97] for a scalar counterexample. However, in our setup, we can anticipate that this question has a positive answer.

The main contribution of this paper is to prove the exponential decay in (2), and consequently the uniform second moment stability for $\mathcal{S}$, by assuming that the parameters $A_{k}$, $k \geq 0$, form an almost periodic sequence (Theorem 3.1). Basic conditions are also required to validate the result, such as the existence of the average cost (1), together with the controllability and observability of the pairs $\left(A_{k}, B_{k}\right)$ and $\left(A_{k}, C_{k}\right)$ (Assumption 2.1).

The almost periodic approach is useful for applications, as illustrated in this paper by a practical application that implements an almost periodic linear state-feedback strategy to control the velocity of a DC motor device (Section V). Motivation for applications of almost periodic parameters is not limited to the case handled in this paper, as one can see in the study of population models [18], in characterizing almost periodic solutions for unidimensional AR models [19], in determining the controllability of nonlinear systems [20], 
in the construction of the fundamental matrix for autonomous systems [21], in the estimation of stochastic processes with almost periodic covariance functions [22], just to cite a few examples. These illustrate some of the effective applications arising from the almost periodic system theory.

The paper is organized as follows. Section II quotes the basic notions of controllability and observability for linear time-varying systems. Section III presents the almost periodic definition and the main result, whereas Section IV is dedicated to prove the main result. Finally, to illustrate the result, we describe in Section $\mathrm{V}$ a strategy to control in practice the velocity of a DC motor apparatus with almost periodic statefeedback structure.

\section{NotATION, DEFINITIONS, AND THE MAIN RESUlT}

We denote respectively the real and natural numbers by $\mathbb{R}$ and $\mathbb{N}$. The normed linear space of all $n \times m$ real matrices is denoted by $\mathbb{R}^{n, m}$. The superscript ' indicates the transpose of a matrix. Let $\mathbb{S}_{+}^{n}$ be the closed convex cone $\left\{U \in \mathbb{R}^{n, n}: U=\right.$ $\left.U^{\prime} \geq 0\right\} ; U \geq V$ signifies that $U-V \in \mathbb{S}_{+}^{n}$. Let $\operatorname{tr}\{\cdot\}$ be the trace operator and $\rho(\cdot)$ be the spectral radius operator. If $U$ is a matrix in $\mathbb{R}^{m, n}$, we let $\|U\|:=\operatorname{tr}\left\{U^{\prime} U\right\}^{\frac{1}{2}}$ be the Euclidean (Frobenius) norm for matrices. We say that a matrix sequence $\left\{U_{k} ; k \geq 0\right\}$ is bounded if $\sup _{k \in \mathbb{N}}\left\|U_{k}\right\|<\infty$.

In association with the matrix sequence $\left\{A_{k} ; k \geq 0\right\}$ in $\mathcal{S}$, we can define a family of discrete evolution operators, namely,

$$
\Phi(k, i)=A_{k-1} A_{k-2} \cdots A_{i}, \quad \text { for each } k>i \geq 0,
$$

with $\Phi(k, k)$ being the identity matrix for each $k \geq 0$. Let us now recall the concepts of uniform controllability and observability, e.g., see [23], [24].

Definition 2.1: The pair $\left(A_{k}, B_{k}\right)$ is uniformly controllable (or simply controllable) if there exists $\tau_{c} \geq 1$ and a real number $\sigma_{c}>0$ such that

$\sum_{i=0}^{\tau_{c}-1} \Phi(k, k-i) B_{k-i-1} B_{k-i-1}^{\prime} \Phi(k, k-i)^{\prime} \geq \sigma_{c} I, \quad \forall k \geq \tau_{c}$.

Definition 2.2: The pair $\left(A_{k}, C_{k}\right)$ is uniformly observable (or simply observable) if there exists $\tau_{0} \geq 1$ and a real number $\sigma_{0}>0$ such that

$$
\sum_{i=0}^{\tau_{0}-1} \Phi(k+i, k)^{\prime} C_{k+i}^{\prime} C_{k+i} \Phi(k+i, k) \geq \sigma_{0} I, \quad \forall k \geq 0 .
$$

Assumption 2.1: (Basic assumption). The pairs $\left(A_{k}, B_{k}\right)$ and $\left(A_{k}, C_{k}\right)$ are controllable and observable, respectively, and the average cost value in (1) exists.

The investigation in the remaining part of this paper will be concentrated on conditions to assure the uniform second moment stability for $\mathcal{S}$.

\section{Almost PERIODIC DEFINITION AND MAIN RESUlT}

Let us recall the Bohr's definition for almost periodic sequences.

Definition 3.1: ([25, p. 153],[4, p. 205]). A function $f$ : $\mathbb{N} \mapsto \mathbb{B}$, which represents a map from the domain set of integers $\mathbb{N}$ into a given Banach space $\mathbb{B}$, is called almost periodic if for any $\varepsilon>0$ there corresponds a natural number $N=N(\varepsilon)$ such that, among any $N$ consecutive integers, there exists at least one integer $p$ with the property

$$
\|f(k+p)-f(k)\|<\varepsilon, \quad \forall k \geq 0 .
$$

The number $p$ is called an $\varepsilon$-translation number of $f(k)$.

Remark 3.1: An important property taken from the almost periodic definition is as follows. For each $\varepsilon>0$, there exists a natural number $N=N(\varepsilon)$ such that in each interval $(i N, \ldots,(i+1) N]$ there occurs an $\varepsilon$-translation number $p_{i}$, or equivalently, one can extract a subsequence $\left\{p_{i}\right\}$ in $\mathbb{N}$ such that

$i N<p_{i} \leq(i+1) N \Rightarrow\left\|f\left(k+p_{i}\right)-f(k)\right\|<\varepsilon, \quad \forall i, k \in \mathbb{N}$.

\section{A. Main result}

In the sequel, we assume that the sequence of parameters $\left\{A_{k}\right\}$ is almost periodic. This condition, accompanied by the existence of the average cost (Assumption 2.1), allows us to assure the second moment stability for the system $\mathcal{S}$; more precisely,

Theorem 3.1: If Assumption 2.1 holds and $\left\{A_{k}\right\}$ is almost periodic, then the system $\mathcal{S}$ is uniformly second moment stable.

The proof of Theorem 3.1 is postponed to Section IV. The next numerical example illustrates the result of Theorem 3.1.

Example 3.1: Let us define the sequence $\left\{a_{k}\right\}$ as follows.

$a_{0}=1, \quad a_{1}=0, \quad a_{2^{n}+k}=a_{k}-\frac{1}{2^{n}}, \quad k=0, \ldots, 2^{n}-1$,

$\forall n \geq 1$. The scalar sequence $\left\{a_{k}\right\}$ is almost periodic, c.f. [26, Lem. 4.1]. Consider in addition the almost periodic sequences $\left\{b_{k}\right\}$ and $\left\{c_{k}\right\}$ defined as

$$
\begin{aligned}
& b_{k}=\frac{1-\exp (-2-\sin \sqrt{2} k)}{3+\sin \sqrt{2} k} \quad \text { and } \\
& c_{k}=\frac{\sin \sqrt{2} k}{1+\ln (2+\cos \sqrt{2} k)}, \quad \forall k \in \mathbb{N} .
\end{aligned}
$$

The parameters of the system $\mathcal{S}$ are defined below:

$$
A_{k}=\left(\begin{array}{cc}
a_{k} & b_{k} \\
0 & c_{k}-b_{k}
\end{array}\right), B_{k}=C_{k}=I, \quad \text { and } D_{k}=0,
$$

$\forall k \in \mathbb{N}$, where $I$ denotes the identity matrix. The matrix sequence $\left\{A_{k}\right\}$ is almost periodic (Fig. 1), and one can easily verify that the pairs $\left(A_{k}, B_{k}\right)$ and $\left(A_{k}, C_{k}\right)$ are controllable and observable, respectively. The numerical evaluation (see Appendix 2) indicates that the long-run average cost (1) is equal to $J=3.0209$, so that the result of Theorem 3.1 assures that the system $\mathcal{S}$ is uniformly second moment stable.

\section{Proof of TheOREM 3.1}

This section presents the main reasoning to prove Theorem 3.1. For this purpose, we present a series of preliminary results before passing to the main argument. 


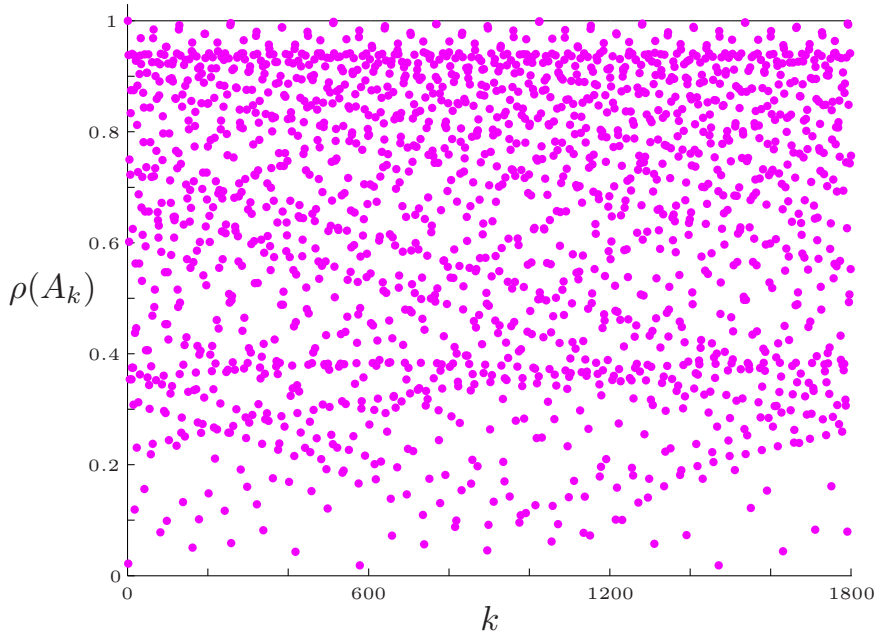

Fig. 1. Spectral radius of the almost periodic sequence $\left\{A_{k}\right\}$ as described in Example 3.1.

Lemma 4.1: Let $\{f(k)\}$ be an almost periodic sequence of non-negative real numbers. If there exist a constant $L>0$ and a strictly increasing subsequence $\left\{d_{k}\right\}$ in $\mathbb{N}$ such that

$$
f\left(d_{k}+\ell\right) \geq L, \quad \forall \ell=0, \ldots, k, \quad \forall k>0,
$$

then $f(k) \geq L$ for all $k \geq 0$.

Proof: Take an arbitrary $\varepsilon_{0}>0$, and following Remark 3.1 there exist a natural number $N_{0}=N\left(\varepsilon_{0}\right)$ and a subsequence $\left\{p_{i}\right\}$ in $\mathbb{N}$ of $\varepsilon_{0}$-translations such that

$i N_{0}<p_{i} \leq(i+1) N_{0} \Rightarrow\left|f\left(k+p_{i}\right)-f(k)\right|<\varepsilon_{0}$,

From the assumption (6), we can choose a constant $k_{0}>$ $2 N_{0}$ such that

$$
f\left(d_{k_{0}}+\ell\right) \geq L, \quad \forall \ell=0, \ldots, k_{0} .
$$

Now, from the choice $k_{0}>2 N_{0}$, one can note that there is some $i_{0}>0$ such that the interval $\left(i_{0} N_{0}, \ldots,\left(i_{0}+2\right) N_{0}\right]$ is contained within the larger interval $\left[d_{k_{0}}, \ldots, d_{k_{0}}+k_{0}\right]$. In particular, it follows from the left-hand side expression of (7) that $\left[p_{i_{0}}, \ldots, p_{i_{0}}+N_{0}\right]$ is a subset of $\left(i_{0} N_{0}, \ldots,\left(i_{0}+2\right) N_{0}\right]$, and hence $\left[p_{i_{0}}, \ldots, p_{i_{0}}+N_{0}\right]$ is contained in the interval $\left[d_{k_{0}}, \ldots, d_{k_{0}}+k_{0}\right]$, so that (8) yields

$$
f\left(p_{i_{0}}+n\right) \geq L, \quad \forall n=0, \ldots, N_{0} .
$$

Combining (7) and (9), we have

$L \leq f\left(p_{i_{0}}+n\right) \leq\left|f\left(p_{i_{0}}+n\right)-f(n)\right|+f(n)<\varepsilon_{0}+f(n)$, which results in

$$
f(n) \geq L-\varepsilon_{0}, \quad \forall n=0, \ldots, N_{0} .
$$

The inequality in (10) applied into (7) allows us to conclude that

$f\left(n+p_{i}\right)>f(n)-\varepsilon_{0} \geq L-2 \varepsilon_{0}, \quad \forall n=0, \ldots, N_{0}, \forall i>0$, which shows the result since $\varepsilon_{0}>0$ was chosen arbitrarily.
Let us now recall some basic facts from almost periodic sequences.

Proposition 4.1: ([25, Th. 6.1, p. 154], [4, Prop. 2, Appendix B]). An almost periodic sequence is bounded.

Proposition 4.2: ([25, Th. 1.5, p. 11]). The sum (product) of two almost periodic sequences results in an almost periodic sequence.

Remark 4.1: If $\left\{A_{k}\right\}$ is almost periodic, then the sequence $\left\{A_{k+n}\right\}$ due to an $n$-th step translation is also almost periodic. Hence, due to Proposition 4.2, the sum of sequences

$$
\left\{A_{k}+A_{k+1} A_{k}+\ldots+A_{k+n} \cdots A_{k}\right\}_{k=0}^{\infty}
$$

is almost periodic whenever $n$ is finite. If we set

$$
g_{n}(k):=\sum_{\ell=1}^{n}\|\Phi(k+\ell, k)\|, \quad \forall n \geq 1, \forall k \geq 0,
$$

then the aforementioned conclusions yield the next result.

Lemma 4.2: If $\left\{A_{k}\right\}$ is almost periodic, and if $n>0$ is fixed and finite, then the sequence $\left\{g_{n}(k)\right\}_{k=0}^{\infty}$ is almost periodic.

The next result is useful in a contradiction argument to be presented later.

Lemma 4.3: Suppose that there exist some $L_{0}>0$ and a strictly increasing sequence $\left\{d_{k}\right\}$ in $\mathbb{N}$ such that

$$
g_{\infty}\left(d_{k}+\ell\right) \geq L_{0}, \quad \forall \ell=0, \ldots, k .
$$

Then, for any given $L<L_{0}$, there exists some $n_{0}>0$ such that

$$
g_{n_{0}}\left(d_{k}+\ell\right)>L, \quad \forall \ell=0, \ldots, k .
$$

The proof of Lemma 4.3 is available in Appendix 1.

Next we present an useful inequality for the quantity $g_{n}(\cdot)$. For this purpose, we need to introduce the number

$$
\alpha:=\sup _{k \in \mathbb{N}}\left\|A_{k}\right\|,
$$

which is finite in view of Proposition 4.1. Without loss of generality, we consider $\alpha \geq 1$.

Regarding the identity $\Phi(k, k-1)=A_{k-1}$ for all $k>0$, we can see from the definition in (11) that

$$
\begin{aligned}
\alpha\left(1+g_{n}(k)\right) & \geq\left\|A_{k-1}\right\|\left(1+g_{n}(k)\right) \\
& =\|\Phi(k, k-1)\|\left(1+\sum_{\ell=1}^{n} \alpha\|\Phi(\ell+k, k)\|\right) \\
& \geq\|\Phi(k, k-1)\|+\cdots+\|\Phi(k+n, k-1)\| .
\end{aligned}
$$

On the other hand, due to the identity

$$
\|\Phi(k, k-1)\|+\cdots+\|\Phi(k-1+n, k-1)\|=g_{n}(k-1),
$$

we get from (14) that $\alpha\left(1+g_{n}(k)\right) \geq g_{n}(k-1)$. Equivalently, we have

$$
g_{n}(k) \geq \frac{g_{n}(k-1)}{\alpha}-1, \quad \forall k \geq 1, \forall n>0 .
$$

The next three results are valid for general time-varying matrix sequences $\left\{A_{k}\right\}$.

Proposition 4.3: ([15, Lem. 3.1]). Let the pair $\left(A_{k}, B_{k}\right)$ be controllable. Then the system $\mathcal{S}$ is uniformly second moment 
stable if and only if there exist real numbers $\gamma \geq 1$ and $0<$ $\xi<1$ such that

$$
\|\Phi(k+\ell, k)\| \leq \gamma \xi^{\ell}, \quad \forall k, \ell \in \mathbb{N} .
$$

Proposition 4.4: ([7, Th. 1]). There exists a real number $L>0$ such that $g_{\infty}(k) \leq L$ for all $k \geq 0$ if and only if there exist real numbers $\gamma \geq 1$ and $0<\xi<1$ such that (16) holds true.

The next result is the key to our subsequent analysis.

Proposition 4.5: ([15, Th. 2.1]). If Assumption 2.1 holds, then there exist a strictly increasing subsequence $\left\{m_{i}\right\}$ in $\mathbb{N}$ and a real number $L>0$ such that $g_{\infty}\left(m_{i}\right) \leq L$ for all $i \in \mathbb{N}$.

Remark 4.2: We want to assure the exponential decay as in (16) to conclude that the stochastic system $\mathcal{S}$ is uniformly second moment stable (Proposition 4.3). Thus, it suffices to assure that $g_{\infty}(k) \leq L$ for all $k \geq 0$ (Proposition 4.4). Proposition 4.5 guarantees the existence of an upper bound for $g_{\infty}\left(m_{i}\right)$ only for a specific subsequence $\left\{m_{i}\right\}$, which does not cover the situation in which $g_{\infty}(k) \leq L$ holds for all $k \geq 0$, see the counterexample in [15, Ex. 3.1]. A contribution of this paper is to show that $g_{\infty}(k) \leq L$ indeed holds true for all $k \geq 0$ when $\left\{A_{k}\right\}$ is almost periodic, and consequently the system $\mathcal{S}$ is uniformly second moment stable in this setup.

Proof of Theorem 3.1 (continued): Our aim here is to present the arguments to show that the stochastic system $\mathcal{S}$ is uniformly second moment stable. To accomplish this goal, we prove the following claim.

Claim 1: There exists a constant $L>0$ such that $g_{\infty}(k) \leq$ $L$ for all $k \geq 0$.

The desired stability result then follows from the Claim 1 and the discussion of Remark 4.2.

The proof of the claim now proceeds using a contradiction argument. Assume on the contrary that the sequence $\left\{g_{\infty}(k)\right\}$ is unbounded. Then $\left\{g_{\infty}(k)\right\}$ has a subsequence that diverges to infinity. Hence, one can choose a subsequence $\left\{g_{\infty}\left(d_{k}\right)\right\}$ from $\left\{g_{\infty}(k)\right\}$ such that

$$
g_{\infty}\left(d_{k}\right) \geq L_{0}\left(\alpha^{k+1}+\alpha^{k}+\ldots+\alpha\right), \quad \forall k \geq 0,
$$

with $L_{0}$ and $L$ being two arbitrary constants satisfying $L_{0}>$ $(\alpha+1) L$, and $\alpha$ being the constant defined in (13). From (15) and (17), we can write

$$
g_{\infty}\left(d_{k}+1\right) \geq \frac{g_{\infty}\left(d_{k}\right)}{\alpha}-1 \geq L_{0}\left(\alpha^{k}+\alpha^{k-1}+\ldots+\alpha\right) .
$$

By induction, it is possible to conclude that

$g_{\infty}\left(d_{k}+\ell\right) \geq L_{0}\left(\alpha^{k+1-\ell}+\alpha^{k-\ell}+\ldots+\alpha\right), \quad \ell=0,1, \ldots, k$.

Notice from (18) that the values $g_{\infty}\left(d_{k}\right), g_{\infty}\left(d_{k}+\right.$ $1), \ldots, g_{\infty}\left(d_{k}+k\right)$ are bounded below by a polynomial with lesser and lesser degree, also generating a descending staircase (Fig. 2). These lower bounds from (18) are important to devise the next argument.

From the lower bounds of (18), we can see in particular that

$$
g_{\infty}\left(d_{k}+\ell\right) \geq L_{0}, \quad \ell=0,1, \ldots, k .
$$

This inequality and Lemma 4.3 allow us to assure the existence of a constant $n_{0}$ such that

$$
g_{n_{0}}\left(d_{k}+\ell\right)>L, \quad \forall \ell=0, \ldots, k .
$$

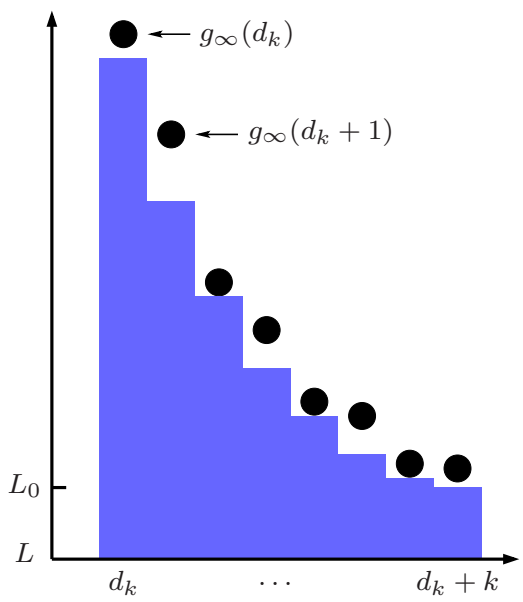

Fig. 2. Illustrative example of lower bounds for the values $g_{\infty}\left(d_{k}\right), \ldots, g_{\infty}\left(d_{k}+k\right)$ driven by the diverging sequence $\left\{g_{\infty}\left(d_{k}\right)\right\}$

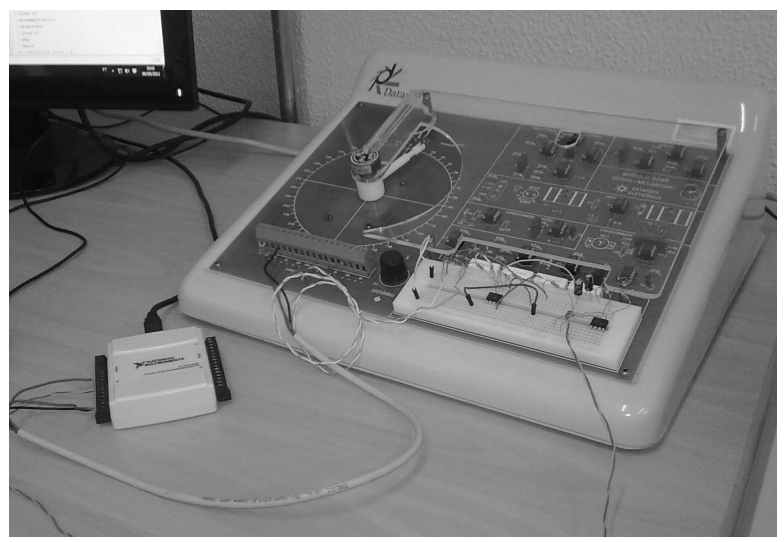

Fig. 3. Laboratory DC motor testbed used to perform the experiments of Section V.

Applying (19) in Lemma 4.1, we have that $g_{n_{0}}(k)>L$ for all $k>0$, but this is an absurd since Proposition 4.5 guarantees that $g_{\infty}(m)$ is bounded from above by $L$ for infinitely many values of $m$. This argument of contradiction proves that Claim 1 is valid, and as a consequence, we obtain the uniform second moment stability for $\mathcal{S}$ via Remark 4.2.

\section{Almost PERIODIC PRACTICAL APPLICATION}

The main aim of this section is to describe the behavior of a DC motor apparatus in the presence of almost periodic linear state-feedback. In a usual DC motor device, the two system variables are (i) the velocity of the rotor and (ii) the electrical current consumed by the motor [27], [28], [29]; these two variables are denoted here by $v_{k}$ and $i_{k}, k \geq 0$, respectively. We also used a National Instruments USB-6008 data acquisition card to set a link between the Matlab software and the DC motor Module 2208, Datapool Eletronica Ltda, Brazil (Fig. 3). The device was configured with sampling with rate of 17.16 milliseconds.

Setting the system state as $z_{k} \equiv\left[\begin{array}{ll}v_{k} & i_{k}\end{array}\right]^{\prime}$, we obtain the model of the DC motor device represented as

$$
z_{k+1}=A_{k} z_{k}+B_{k} w_{k}+r_{k}, \quad z_{0} \in \mathbb{R}^{2}, \quad \forall k \geq 0 .
$$




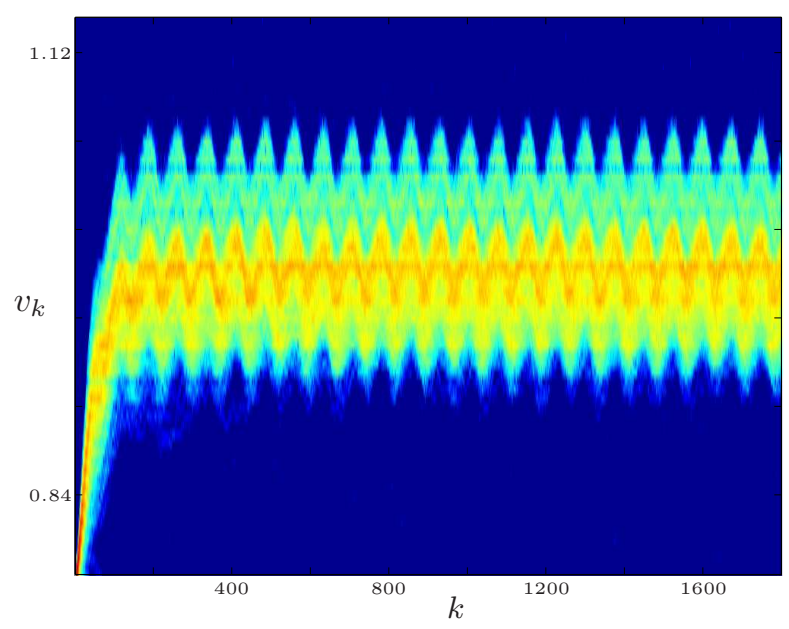

(a) Velocity $(\mathrm{rad} / \mathrm{s})$ of the DC motor device.

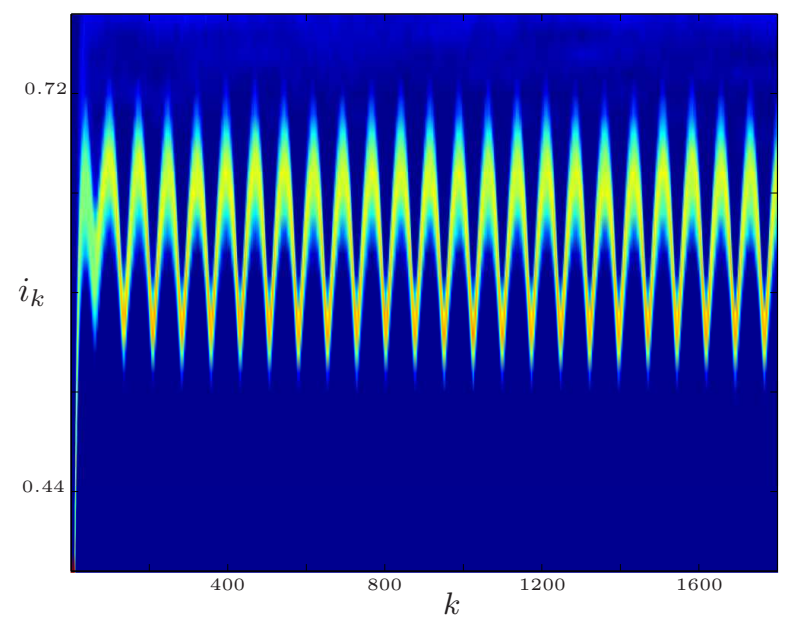

(b) Electrical current, in Ampere, driven by the DC motor device.

Fig. 4. Pictorial representation of the velocity $v_{k}$ and electrical current $i_{k}$ measured from the DC motor device, corresponding to 900 distinct realizations. The figures indicate the almost periodic behavior arising from both variables.

The almost periodic sequences $\left\{G_{1}(k)\right\}$ and $\left\{G_{2}(k)\right\}$ determine the gain in the linear state-feedback configuration and are defined as, for all $k \geq 0$,

$$
\begin{gathered}
G_{1}(k)=0.272+(\sqrt{2} / 100) \ln (2.2 \\
+\sqrt{3} \sin (0.06 \sqrt{2} k-\sqrt{2})) \\
G_{2}(k)=-0.8970+0.001 \sin (0.006 \sqrt{2} k) \cos (0.005 \sqrt{3} k) .
\end{gathered}
$$

The system parameters are

$$
\begin{aligned}
& A_{k}=\left[\begin{array}{ll}
0.938602 & 0.166614 \\
0.109488 & 0.554576
\end{array}\right] \\
& +\left[\begin{array}{c}
0.113355 \\
-0.466798
\end{array}\right]\left[\begin{array}{ll}
G_{1}(k) & G_{2}(k)
\end{array}\right], \quad \forall k \geq 0, \\
& B_{k} \equiv\left[\begin{array}{cc}
1 & 0 \\
0 & 0.5
\end{array}\right] \times 10^{-2}, \quad r_{k} \equiv\left[\begin{array}{c}
-1.06757 \\
3.67366
\end{array}\right] \times 10^{-2} \text {, }
\end{aligned}
$$

and $z_{0}=\left[\begin{array}{ll}0 & 0\end{array}\right]^{\prime}$.

In this experiment, we verify from the theoretical and practical perspectives whether the DC motor device is uniformly central moment stable, i.e., if there exists a constant $c>0$ such that

$$
\mathrm{E}\left[\left\|z_{k}-\mathrm{E}\left[z_{k}\right]\right\|\right] \leq c, \quad \forall k \geq 0 .
$$

In the theoretical analysis, by simply setting $x_{k} \equiv z_{k}-\mathrm{E}\left[z_{k}\right]$ into $\mathcal{S}$, we have that both systems $\mathcal{S}$ and (20) are equivalent with respect to the stochastic analysis [30, Ch. 2]. On that account, the uniform central moment stability for (20) is confirmed by Theorem 3.1, since $\left\{A_{k}\right\}$ is almost periodic and Assumption 2.1 is satisfied with average cost $J=2.2116 \times 10^{-3}$ (see Appendix 2).

In the practical front, we conducted 900 distinct experimental realizations, measuring the velocity and electrical current consumed by the DC motor device. The data obtained in the practical experiments indicates that the mean and standard deviation for both velocity and electrical current are bounded, a fact that suggests that the DC motor device is uniformly central moment stable in practice, see Fig. 4. The data also illustrates the almost periodic behavior arising from the measured velocity and electrical current.

The experiments described in this section emphasize the applicability of the derived approach to characterize the uniform second moment stability for linear stochastic systems with almost periodic parameters.

\section{APPENDiX 1: ProOf of LeMma 4.3}

Before passing to the proof of Lemma 4.3, it is necessary to present the following result.

Lemma 5.1: Suppose that there exist some $L>0$ and a strictly increasing sequence $\left\{m_{i}\right\}$ in $\mathbb{N}$ such that $g_{\infty}\left(m_{i}\right) \geq L$ for all $i \geq 0$. Then for any $\varepsilon>0$ and any strictly increasing sequence $\left\{n_{i}\right\}$ in $\mathbb{N}$, there exists some $i_{0}>0$ such that

$$
i \geq i_{0} \Rightarrow g_{n_{i}}\left(m_{i}\right) \geq L-\varepsilon
$$

Proof: If we deny the assertion in (21), then the inequality

$$
g_{n_{i_{k}}}\left(m_{i_{k}}\right)<L-\varepsilon
$$

holds true for infinitely many values of $i_{k}$, and since $n_{i_{k}} \uparrow \infty$ as $k \rightarrow \infty$, we have

$$
\limsup _{k \rightarrow \infty} g_{n_{i_{k}}}\left(m_{i_{k}}\right)<L-\varepsilon<L \leq g_{\infty}\left(m_{i}\right), \quad \forall i>0,
$$

which is an absurd. This argument proves the result.

Proof of Lemma 4.3 (continued): Since $g_{n}(\cdot)$ is monotone with respect to $n$, and due to the assumption that $L_{0}>L$, we can deduce from (12) that there exists a finite integer $\bar{n}$ such that

$$
g_{\bar{n}}\left(d_{k}+\ell\right) \leq L \quad \text { and } \quad g_{\bar{n}+1}\left(d_{k}+\ell\right)>L .
$$

Let $\bar{n}\left(d_{k}, \ell\right)$ be the $\bar{n}$ value in (22) corresponding to $d_{k}$ for each $\ell=0, \ldots, k$. Define

$$
\bar{n}_{\max }(k)=\max \left\{\bar{n}\left(d_{k}, 0\right), \ldots, \bar{n}\left(d_{k}, k\right)\right\} .
$$

Claim 2: The sequence $\left\{\bar{n}_{\max }(k)\right\}_{k=0}^{\infty}$ is bounded.

Let us assume for the moment that Claim 2 holds true. In this case, by setting the number

$$
n_{0}=1+\max _{k \in \mathbb{N}} \bar{n}_{\max }(k),
$$


we get from the right-hand side of (22) that $g_{n_{0}}\left(d_{k}+\ell\right)>L$ for all $\ell=0, \ldots, k$ and all $k>0$, which is what we wished to show.

The remaining part of this section is dedicated to prove the validity of Claim 2. In fact, if we deny the assertion of Claim 2 , then it is equivalent to allow the existence of a subsequence $\left\{\bar{n}_{\max }\left(k_{i}\right)\right\}$ from $\left\{\bar{n}_{\max }(k)\right\}$ that diverges to infinity. This occurs only if there exists a corresponding sequence $\left\{s_{i}\right\}$ such that

$$
\bar{n}\left(d_{k_{i}}, s_{i}\right) \uparrow \infty \quad \text { as } \quad i \rightarrow \infty .
$$

Notice that $\bar{n}\left(d_{k_{i}}, s_{i}\right)$ belongs to the set $\left\{\bar{n}\left(d_{k_{i}}, 0\right), \ldots, \bar{n}\left(d_{k_{i}}, k_{i}\right)\right\}$, and for this reason $\left\{s_{i}\right\}$ conforms to the rule

$$
0 \leq s_{i} \leq k_{i}, \quad \forall i \geq 0 .
$$

The fact in (24) is important since it assures that the value $d_{k_{i}}+s_{i}$ belongs to the interval $\left[d_{k_{i}}, d_{k_{i}}+k_{i}\right]$. This observation and (12) allow us to write

$$
g_{\infty}\left(d_{k_{i}}+s_{i}\right) \geq L_{0}, \quad \forall i>0 .
$$

Now, we can apply (25) in Lemma 5.1 to assure the existence of some $i_{0}>0$ such that

$$
i \geq i_{0} \Rightarrow g_{\bar{n}\left(d_{k_{i}}, s_{i}\right)}\left(d_{k_{i}}+s_{i}\right) \geq L_{0}-\varepsilon .
$$

The contradiction to prove Claim 2 now arises. Notice that we can invoke the left-hand side of (22) to obtain the inequality

$$
g_{\bar{n}\left(d_{k_{i}}, s_{i}\right)}\left(d_{k_{i}}+s_{i}\right) \leq L, \quad \forall i>0,
$$

which contradicts (26) since $L_{0}>L$ and $\varepsilon>0$ is taken to be sufficiently small. This argument shows the contradiction and proves that the statement in Claim 2 is true.

\section{APPENDIX 2: NUMERICAL EVALUATION OF THE LONG-RUN AVERAGE COST}

Let the second moment matrix of $x_{k}$ and $y_{k}$ be defined, respectively, as

$$
X(k)=\mathrm{E}\left[x_{k} x_{k}^{\prime}\right], \quad \text { and } \quad Y(k)=\mathrm{E}\left[y_{k} y_{k}^{\prime}\right], \quad \forall k \geq 0 .
$$

From [30, Ch. 2, p. 20], we have

$$
\begin{aligned}
X(k+1) & =A_{k} X(k) A_{k}^{\prime}+B_{k} B_{k}^{\prime}, \\
Y(k) & =C_{k} X(k) C_{k}^{\prime}+D_{k} D_{k}^{\prime}, \quad \forall k \geq 0, \quad X(0)=x_{0} x_{0}^{\prime} .
\end{aligned}
$$

In addition, there holds $\mathrm{E}\left[\left\|y_{k}\right\|^{2}\right]=\left\|Y(k)^{\frac{1}{2}}\right\|^{2}$, for all $k \geq 0$, an the long-run average cost is identical to

$$
J=\limsup _{N \rightarrow \infty} \frac{1}{N} \sum_{k=0}^{N-1}\left\|Y(k)^{\frac{1}{2}}\right\|^{2} .
$$

\section{REFERENCES}

[1] H. D. Angelo, Linear Time-Varying Systems: Analysis and Synthesis. Allyn and Bacon, Boston, 1970.

[2] J. L. Willems, Stability Theory of Dynamical Systems. Great Britain: Thomas Nelson and Sons Ltd., 1970.

[3] B. D. O. Anderson and J. B. Moore, "Detectability and stabilizability of discrete-time linear systems," SIAM J. Control Optim., vol. 19, no. 1, pp. 20-32, 1981.

[4] A. Halanay and V. Ionescu, Time-Varying Discrete Linear Systems. Birkhäuser Verlag, Basel, Switzerland, 1994.
[5] W. H. Kwon and A. E. Pearson, "On feedback stabilization of timevarying discrete linear systems," IEEE Trans. Automat. Control, vol. 23, pp. 479-481, 1978.

[6] K. M. Przyluski and S. Rolewicz, "On stability of linear timevarying infinite-dimensional discrete-time systems," Systems Control Lett., vol. 4, no. 5, pp. 307-315, 1984.

[7] C. S. Kubrusly, "Uniform stability for time-varying infinite-dimensional discrete linear systems," IMA J. Math. Control Inform., vol. 5, no. 4, pp. 269-283, 1988.

[8] H. Lin and P. J. Antsaklis, "Stability and stabilizability of switched linear systems: A survey of recent results," IEEE Trans. Automatic Control, no. 2, pp. 308-322.

[9] G. Chesi, A. Garulli, A. Tesi, and A. Vicino, Homogeneous Polynomial Forms for Robustness Analysis of Uncertain Systems. Berlin Germany: Springer, 2009, vol. 390, ch. Series: Lecture Notes in Control and Information Sciences.

[10] F. Kozin, "A survey of stability of stochastic systems," Automatica, vol. 5, pp. 95-112, 1969.

[11] L. Arnold, Stochastic differential equations: Theory and applications. Wiley-Interscience, 1974.

[12] H. J. Kushner, Stochastic stability and control. New York, USA: Academic Press Inc., 1967.

[13] R. Z. Khasminskii, Stochastic Stability of Differential Equations. Sijthoff \& Noordhoff, Alphen aan den Rijn - Germantown, 1980, reprinted in 2012 by Springer-Verlag.

[14] I. Y. Kats and A. A. Martynyuk, Stability and Stabilization of Nonlinear Systems with Random Structures. Abingdon, Oxfordshire, UK: Taylor \& Francis, 2002.

[15] A. N. Vargas and J. B. R. do Val, "Average cost and stability of timevarying linear systems," IEEE Trans. Automat. Control, vol. 55, pp. 714-720, 2010.

[16] C. Conley and R. Miller, "Asymptotic stability without uniform stability: Almost periodic coefficients," J. Differential Equations, vol. 1, pp. 333336, 1965.

[17] A. Fink, Almost Periodic Differential Equations, ser. Lecture Notes in Mathematics. Springer-Verlag, Berlin, 1974, vol. 377.

[18] T. Diagana, S. Elaydi, and Y. Abdul-Aziz, "Population models in almost periodic environments," J. Difference Equ. and Appl., vol. 13, no. 4, pp. 239-260, 2007.

[19] H. Hurd, A. Makagon, and A. G. Miamee, "On AR(1) models with periodic and almost periodic coefficients," Stochastic Process. Appl., vol. 100, no. 1-2, pp. 167-185, 2002.

[20] F. Colonius and T. Wichtrey, "Control systems with almost periodic excitations," SIAM J. Control Optim., vol. 48, no. 2, pp. 1055-1079, 2009.

[21] M. Pinto and G. Robledo, "Cauchy matrix for linear almost periodic systems and some consequences," Nonlinear Anal., vol. 74, no. 16, pp. 5426-5439, 2011.

[22] K.-S. Lii and M. Rosenblatt, "Estimation for almost periodic processes," Ann. Statist., vol. 34, no. 3, pp. 1115-1139, 2006.

[23] J. B. Moore and B. D. O. Anderson, "Coping with singular transition matrices in estimation and control stability theory," Internat. J. Control, vol. 31, no. 3, pp. 571-586, 1980.

[24] L. G. Van Willigenburg and W. De Koning, "Linear systems theory revisited," Automatica, vol. 44, no. 7, pp. 1686-1696, 2008.

[25] C. Corduneanu, Almost Periodic Functions, 2nd ed. New York, N.Y., USA: Chelsea Publishing Company, 1989

[26] M. Vesely, "Construction of almost periodic sequences with given properties," Electron. J. Differential Equations, vol. 1, no. 126, pp. 1-22, 2008.

[27] W. Leonhard, Control of Electrical Drives, 3rd ed. New York: SpringerVerlag, 2001.

[28] A. Rubaai and R. Kotaru, "Online identification and control of a DC motor using learning adaptation of neural networks," IEEE Trans. Industry Appl., vol. 36, no. 3, pp. 935-942, 2000.

[29] A. N. Vargas, E. F. Costa, and J. B. R. do Val, "On the control of Markov jump linear systems with no mode observation: application to a DC motor device," Int. J. Robust Nonlinear Control, vol. 23, no. 10, pp. 1136-115, 2013.

[30] B. D. O. Anderson and J. B. Moore, Optimal Filtering. Prentice-Hall, Englewood Cliffs, N.J., 1979. 